\title{
Post-injection Sciatic Neuropathy: A five-year review of cases managed in a paediatric hospital in Ibadan, Nigeria
}

\author{
Alonge, I.A.O., Akinwola, M.O. \\ Department of Physiotherapy, College of Medicine, University of Ibadan, Nigeria \\ Correspondence \\ Dr. I.A.O. Alonge, Department of Physiotherapy, College of Medicine, University of Ibadan, Nigeria \\ E-mail: ibidee@yahoo.com
}

\begin{abstract}
SUMMARY
The World Health Organization (WHO) reported that the administration of injections is one of the most common healthcare procedures, and unsafe injections are associated with morbidity and mortality, especially in developing countries. Post-injection sciatic neuropathy (PISN) has been identified as a serious complication of gluteal intramuscular (IM) injections. The long-term care of PISN poses an economic burden on carers and increases physiotherapists' burden of care.

This study reviewed two hundred and ten cases of PISN among eight hundred paediatric cases seen from January 2004 to December 2008 in the Physiotherapy Clinic of Oni Memorial Children's Hospital in Ibadan, Nigeria. Each patient was evaluated for the limb affected, the health care centre where the injection was given and the health care personnel who gave the injection.

One hundred and twenty three $(58.6 \%)$ of the patients were male while eighty seven $(41.4 \%)$ were female. A majority of the injections were administered at private hospitals - $143(68.1 \%)$. The others were at general hospitals $22(11.2 \%)$, by 'nurses' at dispensing shops $17(8.1 \%)$, at primary healthcare centres $12(5.7 \%)$, and two $(1 \%)$ were given at home by the children's grandmothers.

Since PISN is a preventable condition, there is a need to minimize gluteal IM injections especially when other routes such as the vastus lateralis muscle can be used. A majority of the children with PISN (84.7\%) were below the age of five and this makes it imperative for caution to be exercised when gluteal IM injections are given to this age group. Update and refresher courses should be regularly organized for the health professionals concerned, to minimize the occurrence of PISN.
\end{abstract}

KEY WORDS: post-injection, sciatic neuropathy, paediatric, intramuscular

\section{INTRODUCTION}

Post-injection sciatic neuropathy (PISN) can be defined as a disorder of the sciatic nerve resulting from the use of an improper technique during, or due to, the introduction of a medicinal substance into the gluteal region. It is also referred to as injection-induced sciatic nerve injury, traumatic injection neuropathy (Mansoor, 2004), or postinjection sciatic nerve injury. A puncture of the sciatic nerve or a blood vessel close to it by an injection needle can directly injure the nerve or cause a haematoma that can lead to compression. An abscess in the vicinity can also cause PISN though with delayed presentation (Mansoor,
2004). Sciatic neuropathy is identified as a serious but preventable complication of gluteal intramuscular (IM) injections (Fatunde and Familusi, 2001). Children are more vulnerable to PISN because of the variation in the thickness of their subcutaneous tissue and the depth of their gluteus musculature (Ahuja, 2003). Sciatic neuropathy, especially in children, manifests by paresis in the distribution of the sciatic nerve, followed by minor to severe transient sensory disturbance - causalgia or burning pain in the extremity several hours or days later (Mayer and Romain, 2001). The diagnosis of sciatic palsy may be delayed for up to an average of 3.8 months after the gluteal 
IM injection (Fatunde and Familusi, 2001; Ahuja, 2003, Mansoor, 2004). Affected individuals usually present with foot drop and this can result in varying degrees of motor disability depending on the timing and duration of corrective measures instituted (Oyedeji et al., 2006).

Children with foot drop were previously thought to have had 'missed' cases of polio when in fact they had suffered injuries to their sciatic nerves with gluteal IM injections given in infancy (Nwuga and Odunowo, 1978). The most reported causes of PISN are: unnecessary injections, and faulty techniques in the administration of injections by unqualified personnel (Fatunde and Familusi, 2001; Oyedeji et al., 2006). The W orld Health Organization (WHO) in 1999 reported that out of the 8-12 billion injections administered worldwide annually, the vast majority (90-95\%) are for therapeutic purposes, 50 per cent are unsafe, and 75 per cent are unnecessary (Miller and Pisani, 1999; Halsey, 2003). Injection administration is seen as one of the most common healthcare procedures, and unsafe injections are associated with morbidity and mortality, especially in developing countries (Hutin and Chen, 1999; Eser et al., 2009).

This five- year review was carried out in a secondary healthcare institution that attends only paediatric cases in order to investigate the prevalence of PISN in Nigerian children. It is thus hoped that the research will influence the policy makers to institute policies that could lead to the prevention of this problem.

\section{MATERIALS AND METHODS}

The study was carried out at the Oni Memorial Children's Hospital $(\mathrm{OMCH})$, Ibadan, Oyo State, Nigeria. This hospital in the south west of Nigeria was established in 1985 as a secondary health institution, and is the only state government owned children's hospital which provides health care services exclusively for children 12 years and below in Oyo State. The procedure for this review involved a retrieval of the case notes of the patients managed in the physiotherapy department of the hospital between January 2004 and December 2008, from which the cases of postinjection sciatic neuropathy were identified and reviewed. Information obtained from the case notes was then entered into a spreadsheet specifically designed for the study: and included: hospital number, sex, age, limb affected, personnel by whom, and place where, injection was administered, and physician and physiotherapist's diagnoses. The data were analysed using descriptive statistics of percentages, and the frequency distributions were presented in tables.

\section{RESULTS}

The total number of cases seen at the physiotherapy clinic during the years under review was 800 , out of which there were $210(26.3 \%)$ cases of PISN. Table 1 shows the age distribution pattern of the patients.

Table 1. Age distribution pattern of the patients

\begin{tabular}{ccc}
\hline Age Group (years) & Frequency & Percentage \\
\hline$<1$ & 24 & 11.4 \\
$1-5$ & 154 & 73.3 \\
$>5$ & 32 & 15.2 \\
\hline Total & 210 & 100.0 \\
\hline
\end{tabular}

One hundred and twenty three $(58.6 \%)$ of the patients were male while eighty seven $(41.4 \%)$ were female. Sex distribution according to limb affected is shown in table 2.

Table 2. Sex distribution according to limb affected

\begin{tabular}{lcccc}
\hline \multirow{2}{*}{ Gender } & \multicolumn{4}{c}{ Limb affected } \\
\cline { 2 - 5 } & Right & Left & Both & Total \\
\hline Male & $39(31.7 \%)$ & $82(66.7 \%)$ & $2(1.6 \%)$ & 123 \\
Female & $38(43.7 \%)$ & $48(55.2 \%)$ & $1(1.1 \%)$ & 87 \\
Total & $77(36.7 \%)$ & $130(61.9 \%)$ & $3(1.4 \%)$ & 210 \\
\hline
\end{tabular}

More than half of the injections - $143(68.1 \%)$ - were administered at private hospitals, while two (1\%) were given at home by grandmothers, $17(8.1 \%)$ by 'nurses' at dispensing shops, $12(5.7 \%)$ at primary healthcare centres, and $22(11.2 \%)$ at general hospitals. The records did not show where 13 of the PISN cases were injected. Details of the administered substances were also omitted.

\section{DISCUSSION}

From January 2004 to December 2008, a total of 210 cases of post injection sciatic neuropathy (PISN) attended the Department of Physiotherapy at the Oni Memorial Children's Hospital for treatment. The number observed in this review of paediatric cases is less than the 313 noted by Ezeukwu (2007) but more than the 95 cases recorded by Hamzat and Omotade (2006) and the 27 cases gathered over a period of 12 years by Fatunde and Familusi (2001). This shows the continued occurrence of PISN despite the current advances in medical practice. In most of the cases, 
only one lower limb was affected. This is similar to the results obtained in the studies mentioned above. It is interesting however to note that the three recorded bilateral cases of PISN were after admission at one general and two private health facilities. Children below the age of five constituted more than two thirds $(84.7 \%)$ of the cases seen in this study. This group of children are known to be the most vulnerable to infections and thus, the attendant high mortality in the developing world (Lagunju and Okafor, 2009). Children within age group one to five $(73.3 \%)$ were the ones most affected by PISN in this review, and males $(58.6 \%)$ were more than females. These findings are similar to those obtained in the study by Orubuloye and Ajakaiye (2001). In Nigeria, children are at high risk of contracting malaria, a disease endemic in the studied region. The other four major diseases that affect children in the south western part of Nigeria are measles, typhoid fever, diarrhoea, and respiratory tract infections. Mothers are known to frequent health facilities for the treatment of their children for the above listed and other childhood diseases. The frequency of these diseases and the deaths that occur are so frightening that mothers seek what they believe to be the most effective treatment available at the slightest appearance of any of the symptoms of these diseases (Orubuloye and Ajakaiye, 2001).

The gluteal intramuscular (IM) injection was observed to be a common procedure employed in the care of children (Ndiaye, 2004). It has been suggested that gluteal intramuscular injections should be avoided in children in the vulnerable age group one to five to reduce the occurrence of PISN. The upper part of the lateral thigh (vastus lateralis muscle) could be used instead (Rodger and King, 2000). In the course of the authors' clinical practice, there have been suggestions that it could be the preferred injection site in all age groups.

The highest percentage of injections was administered at private hospitals $(68.1 \%)$. This shows that a high proportion of people in the south western part of the country prefer to patronize private rather than government hospitals. This is in agreement with the reported healthseeking behaviour of people of the south-west region of Nigeria as reported by Orubuloye and Ajakaiye (2001). They found that the larger proportion of women in both urban and rural areas sought treatment for their children in private hospitals, followed by government health facilities, then self-medication and lastly, chemists/ medicine stores. The reasons given for patronizing private hospitals included better service, granting of credit facilities to regular and reliable customers, and accessibility (closer to home). In addition, the service provider could be a relation, or it could be a family decision. Many patients demanded injections on getting to such facilities because of the belief that they provide the best solution to their health problem. Mansoor (2004) alluded to this fact by noting that injections have become a modern magic, with a high demand from patients and the attendant high use among health professionals. This study has further exposed the danger associated with this practice. Members of the public need to be enlightened on the advantages of using other routes of drug administration.

The cost of caring for PISN imposes a burden on the parents of affected children; many therefore default before completing the course of treatment. This could be a reason why there was no clear discharge pattern in the records reviewed.

Functional disability associated with PISN can be devastating. The large percentage of patients with this condition imposes a burden on health care as affected children require intensive rehabilitation procedures by physiotherapists for up to two years, depending on the degree of damage, before there is appreciable improvement (Tak et al., 2008).

It appears that most of the injections were administered by nurses. It is however possible that some of the personnel described as nurses were auxiliaries or other health attendants who, from the observation of these authors, are the personnel sometimes trained by doctors to serve as nurses in many private hospitals because of the proprietors' unwillingness to pay professional nurses. The government thus needs to enact and enforce policies that will ensure that only qualified and licensed nurses are employed by private hospitals. This and other strategies aimed at stamping out quacks from medical practice should be put in place. Compulsory update and refresher courses for all health service staff should also be regularly organized by their various institutions. These would help all concerned to keep abreast of current advances in medical practice.

\section{Acknowledgement}

The authors appreciate the cooperation of the physiotherapists at the Oni Memorial Children's Hospital $(\mathrm{OMCH})$, Ibadan and the medical records officers of the department. 


\section{References}

Ahuja, B. 2003. Post injection sciatic nerve injury. Indian Pediatrics 21: 797-801.

Eser, F., L.A. Aktekin, H. Bodur and C. Atan. 2009. Etiological factors of traumatic peripheral nerve injuries. Neurology India 57: 434-7.

Ezeukwu, A.O. 2007. Injection-induced sciatic nerve injury among children managed in a Nigerian physiotherapy clinic: A five-year review. Journal of Medicine and Rehabilitation 1(1): 22-24.

Fatunde, O.J. and J.B. Familusi. 2001. Injection-induced sciatic nerve injury in Nigerian children. Central Africa Journal of Medicine 47(2): 35-38.

Halsey, N.A. 2003. Commentary: Poliomyelitis and unnecessary injections. International Journal of Epidemiology 32: 278-9.

Hamzat, T.K. and T.T. Omotade. 2006. Acute flaccid paralysis: A five year review of cases managed by physiotherapy at the University College Hospital, Ibadan. African Journal of Health Sciences 13: 28-32.

Hutin, Y.J.F. and R.T. Chen. 1999. Injection safety: A global challenge. Bulletin of the World Health Organization 77: 787788.

Lagunju, I.A. and O.O. Okafor. 2009 An Analysis of Disorders seen at the Paediatric Neurology Clinic, University College Hospital, Ibadan, Nigeria. West African Journal of Medicine 28(1): 326-330.

Mansoor, F. 2004. Case definition of traumatic injection neuropathy. Pakistan Journal of Medical Research 43(3): 1439.
Mayer, M. and O. Romain. 2001. Sciatic nerve paralysis after a buttock intramuscular injection in children: An ongoing risk factor. Archives of Paediatrics 8: 321-3.

Miller, M.A. and E. Pisani. 1999. The cost of unsafe injections. Bulletin of the World Health Organization 77 (10): 808-810.

Ndiaye, A., Y. Sakho, F. Fall, A. Dia, and M.L. Sow. 2004. Sciatic nerve in gluteal portion: application of sciatic nerve post injection lesion. Morphologie 88(282): 135-138.

Nwuga, V.C. and T. Odunowo, 1978. Some clinical characteristics of children with paralytic poliomyelitis referred for physiotherapy. Journal of Tropical Medical Hygiene 81: 84-7.

Orubuloye, I.O. and D.O. Ajakaiye. 2002. Health Seeking Behaviour in Nigeria. New World Press, Ibadan. 80,133.

Oyedeji, O.A., P.O. Elimile, O.J. Adebami, J.D. Ogunlusi, G.A. Oyedeji and S. Oluwafemi. 2006. Injection-induced sciatic nerve injuries among children seen at a Nigerian Physiotherapy unit. The Internet Journal of Third World Medicine 3, 2.

Rodger, M. and L. King. 2000. Drawing up and administering intramuscular injections: A review of the literature. Journal of Advanced Nursing 31 (3): 574-582.

Tak, S.R., N. Dar, M.A. Halwai and M.R. Mir. 2008. Postinjection nerve injuries in Kashmir: A menace overlooked. Journal of Research in Medical Sciences 13 (5): 244-247. 